\title{
How Can the Microenvironment Impair Cancer Immunotherapy? Monoclonal Antibodies and Novel Checkpoint Inhibitors for Cancer Immunotherapy
}

\author{
*Jorge Augusto Borin Scutti \\ Department of Immunology, Anderson Cancer Center, USA
}

Submission: May 14, 2017 ; Published: May 24, 2017

"Correspondence Address: Jorge Augusto Borin Scutti, Department of Immunology, Anderson Cancer Center, Immunotherapy Platform, Houston, Texas, USA, Email: jascutti@mdanderson.org

Abstract

A plenty of studies have investigated the immune system of cancer patients and they suffer from a large immunosuppression mainly due to decrease of lymphocyte proliferation and cytotoxic activity. Several efforts have been made in developing drugs or strategies to improve the treatment of cancer patients. Cancer immunotherapy is one of the best strategy to restore the immune system of cancer patients in attempt to stimulate it to reject and destroy cancer cells. This brief contribution raises the recent findings on immune checkpoint function and the use of checkpoints blockade on cancer immunotherapy.

Keywords: Cancer immunotherapy; Tumor microenvironment (TME); Monoclonal antibodies; Immune checkpoints blockade

\section{Opinion}

Monoclonal antibodies have impacted considerably the care of patients with cancer in the last 30 years. The first report showing the monoclonal antibody technology came out from an article published by Kohler and Milstein in 1975 and caused a tremendous impact on laboratory research. Sometime later Kohler and Milstein would be laureates to the Nobel Prize in Physiology or Medicine in 1984. In 1982 Miller and colleagues using a murine monoclonal antibody against CD20, a protein highly expressed on human B cell lymphomas but poorly expressed on normal B cells to the treatment of 18 lymphoma patients. As result the therapy resulted an objective response of $67 \%$ with minimal toxicity and one patient persisted in complete remission for 72 months and survived for 17 years [1-3].

Over the past decades monoclonal antibodies have aimed proteins on cancer cells such as CD20 (Rituximab, Ofatumumab and Obinutuzumab) and HER-2 (Trastuzumab and Pertuzumab), anti cytokines such anti-IL-6 (Siltuximab), anti-EGFR (Cetuximab and Panitumumab), antiganglioside-G2 (Dinutuximab), antivascular (Bevacizumab and Ramucirumab) or immune cells surface such as anti-CTLA-4 (Ipilimumab and Tremelimumab), anti-PD-1 (Nivolumab and Pembrolizumab), anti-PDL1 (Atezolizumab, Avelumab and Durvalumab). In order to improve the cytotoxicity against cancer cells and induce greater immune responses, monoclonal antibodies have been modified, leaping over some obstacles that could at a first moment reduce the efficacy of its therapy such antigenic modulation, heterogeneity of antigen expression and the delivery of antibodies to tumor cells [4-5]. Furthermore, monoclonal antibodies have been linked to cytotoxic drugs known as monoclonal antibody drug conjugates (ADCs). Some examples of ADCs are Gentuzumab ozogamicin (anti-CD33 conjugated with calicheamicin), Adotrastuzumab (anti-HER2 conjugated with emtansine) and Brentuximab vedotin (anti-CD30 conjugated with vedotin); radionuclides (Y-ibritumomab and 131I-tositumomab) and immunotoxins (Denileukin diftitox, Moxetumomab pasudotox, SL-401, DT2219 and Resimmune) [6].

The immune system can recognize and destroy tumor cells in a process called cancer immunosurveillance. After a century of scientific controversy, the notion that the immune system contributes to cancer development is experiencing a new resurgence - cancer might be seen as a failure of immune surveillance. Recent evidences suggest that the mechanism of tolerance that commonly exist to avoid autoimmune disease may also preclude the development of a proper anti-tumor response and tumors themselves have the competence to antagonize the development of effective immune response against their antigens. Thus, the major challenge has been to develop strategies to breaking this tolerance and identify antigens that are unique to transformed cells [7] 
Advances in our discerning of antigen presentation and tolerance have conduct to some promising strategies. Tumor cells are not just a provincial mass of proliferating abnormal cells, but they are defined as a heterogeneous and structurally complex tissue. These cells can recruit diversity of cell types, including endothelial cells, fibroblasts and immune cells, and, through production and secretion of stimulatory growth factors. This collection of cells and molecules together compose the tumor microenvironment (TME). The microenvironment plays a major role during the initiation and development of tumor progression. During tumor development monocytes and macrophages are actively recruited into tumors where they change the tumor microenvironment to accelerate tumor progression. Several researchers had been showed that distinct microenvironments where tumor-associated macrophages (TAM) promote cancer cell motility, angiogenesis and metastasis. In addition, there is strong evidence that regulatory $\mathrm{T}$ cell populations (Treg) can migrate into tumors and suppress adequate anti-tumor responses in the tumor microenvironment, thus contributing to the prosperity and growth of human tumors. Reasons for limited immune response against tumor cells include immune regulation mediated by cancer cells and immune cells profile on microenvironment [8].

The potential use of immunotherapy is to restore the immune system of patients in attempt to stimulate it to reject and destroy tumors. There are some strategies to modulate the microenvironment such targeting regulatory cells, blocking differentiation or recruitment, blocking immunosuppressive enzymes, regulatory cell depletion, re-programming immunosuppressive cells, modifying the chemokine and cytokine profile and blocking some immune checkpoints [9].

Immune responses are strongly regulated by an amazing system of checkpoints that control positively or negatively the magnitude in a wide range of the immune response. The presence of several checkpoints on T cells can promote activation of naive T cells - effector, memory and regulatory (stimulatory checkpoint pathway) or inhibit the $\mathrm{T}$ cell activation limiting the threshold for $\mathrm{T}$ cell activation and duration of immune response (inhibitory checkpoint pathway) regulating the magnitude of inflammation, tolerance and homeostasis [10].

In 2011, The Food and Drug Administration (FDA) approved the first antibody against the immune checkpoint, an inhibitory molecule called CTLA-4 (Ipilimumab) for the treatment of metastatic melanoma. To date, it is undergoing clinical trials for the treatment of non-small cell lung carcinoma (NSCLC), small cell lung cancer (SCLC), bladder and metastatic hormone refractory prostate cancer. Antibodies against PD-1 (Pembrolizumab and Nivolumab) were approved in 2014 by FDA for the treatment of melanoma patients that did not respond to prior treatment.

This type of therapy symbolizes an innovative concept in cancer therapy due two ways: first, these drugs totally ignore the tumor cells - they reliant on the immune system and second, they are not used to activate the immune system against a particular cancer; they remove inhibitory molecules that block a successful antitumor T cell response. Biologically tumor cells are regarded as poor stimulators of T cells [11]. Immunotherapy records a crucial moment in cancer as long sought attempt to promote the immune system against tumors. The standard treatments for patients with several cancer types are in most cases, surgery, radiation and chemotherapy [12]. The goal of cancer immunotherapy is to reawaken the immune system enabling it to once again attack cancer cells. The basic principles that orchestrate cancer immunology and cancer immunotherapy are immune surveillance, immune editing and immune tolerance [13].

Since anti CTLA-4 and anti PD-1 arrived on the scene, a number of other inhibitory molecules such TIGIT, TIM-3, LAG3 and VISTA have gathered researcher's attention. As discussed above, the immunotherapy combinations with anti-CTLA-4 and anti-PD-1 are bringing tons of hope against several types of cancer. Presently, several efforts have been doing to design reasonable combinations of immunotherapeutic and new agents that target particular pathways to reach synergistic in inhibiting tumor growth and development.

After a plenty of clinical trials and preclinical models, it is clear now that several inhibitory receptors may need to be blocked in order to produce fully $\mathrm{T}$ cell activation and antitumor immunity. Blocking some inhibitory receptors involved in inhibited T cells responses such TIM-3 (T cell immunoglobulin mucin domain 3), LAG-3 (lymphocyte-activation gene 3), TIGIT (T cell immunoglobulin and ITIM domain), BTLA-4 (B and T lymphocyte attenuator), VISTA (immunoglobulin suppressor of $\mathrm{T}$ cell activation) and B7-H4 have emerged as new targets for immunochepoint blockade strategies. In another hand, inducing $\mathrm{T}$ cell activation in stimulating co-stimulatory molecules by monoclonal antibodies such CD27, CD28, ICOS, OX-40, 4-1BB and GITR have been done successfully as cancer immunotherapy strategy against several types of cancer [14].

\section{Conclusion}

The recent approvals by FDA of immune checkpoint antibodies blocking CTLA- 4 and PD- 1 proteins and their power effects on cytotoxic $\mathrm{T}$ lymphocytes in the control of tumors progression and inducing durable therapeutic responses in certain cancer patients has become immune checkpoint targeting a new paradigm for cancer treatment. Rather than targeting the tumor cell, this approach targets molecules on immune cells that regulate their activity to sustain immune responses to cancer and achieve elimination of tumors and immunity to recurrence. This strategy has proven effective in treating many different types of cancer and is now the standard of care for metastatic melanoma and non-small-cell lung cancer (NSCLC). The attractiveness of monoclonal antibodies, especially checkpoints blockades associated to the increasing of anticancer molecules, the improvement of preclinical studies and the advent of screening 


\section{Cancer therapy \& Oncology International Journal}

techniques, there are now not only an unique opportunity to comprehend and overthrow drug resistance through the clinical evaluation of reasonable drug combinations but also allow us to investigate deeply the use of predictive biomarkers to enable patient to respond to several immunotherapeutic agents.

\section{Conflict of Interest}

No potential conflicts of interest were disclosed.

\section{References}

1. Miller RA, Maloney DG, Warnke R, Levy R (1982) Treatment of B-cell lymphoma with monoclonal anti-idiotype antibody. New England Journal of Medicine 306(9): 517-522.

2. Kosmas C, Stamatopoulos N, Stavroyianni N, Tsavaris N, Papadaki T (2002) Anti CD-20=based therapy of B cell lymphoma: state of the art. Leukemia 16 (10): 2004-2015

3. Maloney DG (2012) Anti CD-20 antibody therapy for B-cell lymphomas. N Engl J Med 366(21): 2008-2016.

4. Scott AM, Wolchok JD, Old LJ (2012) Antibody therapy of cancer. Nature Reviews Cancer 12: 278-287.

5. Yan L, Ehrlich PJ, Gibson R, Pickett C, Beckman RA (2009) How can we improve antibody-based cancer therapy? MAbs 1(1): 67-70
6. Bast RC, Carlo M Croce, William N Hait, Waun Ki Hong, Donald W Kufe, et al (2017) Holland Frei Cancer Medicine ( $9^{\text {th }}$ edn). Wiley Blackwell, New Jesery, USA.

7. Schumacher TN, Schreiber RD (2015) Neoantigen in cancer immunotherapy. Science 348 (6230): 69-73.

8. Church SE, Galon J (2015) Tumor microenvironment and immunotherapy: The whole picture is better than a glimpse. Immunity 43(4): 631-633.

9. Rosenberg SA (1999) A new era for cancer immunotherapy based on the genes that encode cancer antigens. Immunity 10(3): 281-287.

10. Sharpe AH (2017) Introduction to checkpoint inhibitors and cancer immunotherapy. Immunological Reviews 276(1): 5-8.

11. Larkin J, Hodi FS, Wolchok JD (2015) Combined Nivolumab and Ipilimumab or Monotherapy in Untreated Melanoma. New Engl J Med 373(13): 23-34

12. Couzin Frankel J (2013) Cancer Immunotherapy. Science 342(6165): 1432-1433.

13. Dunn GP, Bruce AT, Ikeda H, Old LJ, Schreiber RD (2002) Cancer immunoediting: from immunosurveillance to tumor escape. Nat Immunol 3(11): 991-998.

14. Pardol DM (2012) The blockade of immune checkpoints in cancer immunotherapy. Nat Rev Cancer 12(4): 252-264.

\section{Your next submission with Juniper Publishers} will reach you the below assets

- Quality Editorial service

- Swift Peer Review

- Reprints availability

- E-prints Service

- Manuscript Podcast for convenient understanding

- Global attainment for your research

- Manuscript accessibility in different formats

( Pdf, E-pub, Full Text, Audio)

- Unceasing customer service

Track the below URL for one-step submission https://juniperpublishers.com/online-submission.php 